\title{
El Aula Virtual como Herramienta para aumentar el Grado de Satisfacción en el Aprendizaje de las Matemáticas
}

\author{
Lolita González-Hernández ${ }^{1}$ y 2 . \\ (1) Secretaria de Educación Departamental de Caldas. Institución Educativa Neira, Carrera 6 calle 5, Neira, \\ Colombia. \\ (2) Universidad Nacional de Colombia, Sede Manizales, Facultad de Ciencias exactas y naturales, \\ Maestría en enseñanza de las ciencias exactas y naturales, Campus la Nubia, Manizales, CP 170001, \\ Colombia. (e-mail: Igonzalezh@unal.edu.co)
}

Recibido Mar. 23, 2018; Aceptado May. 29, 2018; Versión final Ago. 16, 2018, Publicado Feb. 2019

\begin{abstract}
Resumen
El presente trabajo propone diseñar e implementar un aula virtual para la enseñanza de las matemáticas en alumnos de tercer grado en la Institución Educativa Neira (Colombia), como herramienta para el fortalecimiento de la función neurocognitiva del automonitoreo. Se espera así aumentar el grado de satisfacción de los estudiantes frente a las matemáticas. El desarrollo metodológico parte del análisis de una encuesta tipo escala de Likert y dos test de ideas previas aplicados a los estudiantes, en los cuales se identifican sus principales fortalezas y debilidades. La implementación del aula virtual se centra en la identificación y corrección de los errores propios a partir de la observación de los ejercicios resueltos. El análisis de los resultados obtenidos a partir de la aplicación de la encuesta final de satisfacción, concluye que la implementación del aula virtual aumentó en un $36 \%$ el grado de satisfacción de los estudiantes frente al estudio de las matemáticas.
\end{abstract}

Palabras clave: grado de satisfacción; automonitoreo; aula virtual; test de ideas previas; escala de Likert; aprendizaje matemáticas

\section{The Virtual Classroom as a Tool for Increasing Satisfaction Levels in the Study of Mathematics}

\begin{abstract}
The present investigation proposes the design and implementation of a virtual classroom for mathematics instruction for third grade students at the Institución Educativa Neira (Colombia). This virtual classroom is used as a tool for strengthening the neurocognitive self-monitoring function. In this way it is expected to increase student satisfaction levels in regards to mathematics. The methodological development included analysis of a Likert scale survey and two preconceived notion tests, which were applied to students. From these, their principal strengths and weaknesses were identified. Implementation of the virtual classroom focused on student identification and correction of their own errors, through observation of solved problems. An analysis of the results obtained from application of the final satisfaction survey concludes that implementation of the virtual classroom increased student satisfaction levels in math study by $36 \%$.
\end{abstract}

Keywords: satisfaction levels; self-monitoring; virtual classroom; preconceived notion tests; Likert scale; mathematics study 


\section{INTRODUCCION}

Actualmente, el bajo rendimiento escolar es una problemática extendida: la falta de acompañamiento familiar (Hernández et al., 2017), los problemas de atención y la escasa motivación hacia las actividades académicas (Contreras et al., 2008) han hecho del fracaso escolar un problema común y una de las grandes preocupaciones del sistema educativo. La Institución Educativa Neira no es ajena a estas dificultades y continuamente busca mecanismos y estrategias que fortalezcan los procesos de enseñanza-aprendizaje de acuerdo a la ruta trazada por el Ministerio de Educación Nacional (2012) para el desarrollo de las competencias básicas en matemáticas y lenguaje.

En este orden de ideas y en el caso particular de las matemáticas, siempre se ha tenido presente que su enseñanza es uno de los problemas más difíciles de abordar en las instituciones educativas de básica primaria, debido a que es una de las asignaturas peor comprendidas por los estudiantes y en la cual frecuentemente se obtienen bajos rendimientos académicos (Bishop, 1999). Y al mismo tiempo su aprendizaje se considera fundamental dentro de los procesos académicos. La principal causa de esta situación es la actitud con la que la mayoría de educandos la enfrentan: con la idea generalizada que para su entendimiento y comprensión se requiere un intelecto superior, esta condición genera inseguridad, reduce la motivación y disminuye el grado de satisfacción de los estudiantes hacia el estudio de las matemáticas (Farias y Pérez, 2010).

La revisión de la literatura muestra múltiples teorías de la pedagogía y la psicología que han intentado explicar la naturaleza de la cognición y del comportamiento humano ( Julisa, 2014; Binotti et al., 2009; Martin, 2008; Shulman, 1987). Históricamente han existido diferentes posiciones frente a la relación existente entre el desarrollo de cada individuo y su aprendizaje, Vygostsky (1978a), en un intento por armonizarlas, expone que el aprendizaje es parte importante del desarrollo y asegura que sobre él influye directamente el grado de maduración del sistema nervioso. Tomasello et al. (1993) sostienen que establecer la atención y representar algo en la mente de los demás son requisitos previos necesarios para el proceso de enseñanza y, por esta razón los profesores necesitan conocer la teoría de la mente, con el fin de identificar la necesidad de enseñar y averiguar qué es lo que deben enseñar, para adaptarlo al nivel de habilidad del alumno (Kline, 2015). Adicional a lo anterior, se tiene que el reto actual de las ciencias de la educación es integrar el desarrollo estructural y funcional del cerebro a las investigaciones relacionadas con el aprendizaje, con el fin de comprender en que forma aprenden las personas. $Y$ en el caso particular de la educación matemática, se debe entender como un complejo multidisciplinario, que además de cálculos y operaciones, incluye el contexto social (Ernest, 2016) y la enseñanza-aprendizaje de las Tecnologías de la Información y la Comunicación como herramienta metodológica que potencia el aprovechamiento de temas teóricos en diferentes disciplinas (Televantou et al., 2015).

Así mismo, está presente la importancia del aprendizaje autorregulado, que se refiere a la activación y el sostenimiento de procesos cognitivos, afectivos y comportamentales sistemáticamente orientados hacia el logro de objetivos personales (Zimmerman y Schunk, 2017) y de la metacognición, entendida como el hecho de aprender a aprender, es decir, ser consciente del propio aprendizaje. Por lo anterior, en el desarrollo de esta investigación se incluyen conceptos asociados con la neurociencia, como lo son las funciones cognitivas superiores, consideradas como las facultades mentales de mayor orden a las que se puede llegar mediante el aprendizaje (Julisa, 2014), encargadas de planificar procesos y ejecutar conductas y acciones simples, dirigiéndolas hacia un objetivo concreto más complejo (Correa et al., 2017; Tirapu-Ustárroz, J. et al., 2002), así como de "regular la conducta cognitiva, emocional y social del sujeto" (Sastre-Riba, 2006, p. 143). Es primordial entonces, reconocer el valor que tiene comprender la forma en que piensa y aprende el estudiante y el hecho que su aprendizaje esté principalmente determinado por la motivación (Melo-Solarte y Díaz, 2018), la autovaloración y el grado de satisfacción (Villarruel, 2009), esta última variable influye directamente en el rendimiento académico y en la calidad educativa (Ramírez-Díaz, 2018).

Por otro lado, es necesario tener presente que la actual disponibilidad y rapidez de acceso a las grandes cantidades de información impacta significativamente en el esquema social, así como en sus prioridades y preferencias. La educación como eje fundamental de la sociedad y la cultura no puede ser ajena a estos cambios y se ha visto obligada a renovar sus escenarios y estrategias, cambiando el rol que tradicionalmente ocupan docentes y estudiantes (Falco, 2017), reenfocándose en la resolución de problemas y usando las TIC como herramienta para enriquecer la didáctica, no solo capturando la atención de los estudiantes y divirtiéndolos, sino también resignificando el aula con contenidos contextualizados que los inviten a leer y a transformar su realidad próxima (Castro, 2005), utilizando la motivación y la intención de aprender de los estudiantes como estimulantes de su propio desarrollo (Vygotsky, 1978b) y considerando las diferencias en los presaberes, las personalidades y los estilos de aprendizaje de los estudiantes (Cohen y Baruth, 2017). 
Entre los recursos TIC, que a partir de su integración con la pedagogía conllevan a aprendizajes significativos, resaltan las aulas virtuales (Basantes et al., 2017), las cuales favorecen el aprendizaje autónomo, propio de la corriente pedagógica constructivista, (Esteban-Albert y Zapata-Ros, 2016) a la vez que amplían la cobertura del curso y facilitan la construcción de actividades interactivas ajustadas a los intereses del grupo objetivo, así como la inserción de contenidos multimedia existentes y el registro sistematizado de los procesos individuales. De igual forma el uso de herramientas informáticas incentiva a las nuevas generaciones a utilizar las TIC con propósitos educativos.

El objetivo del presente trabajo es diseñar y aplicar un aula virtual para la enseñanza de las matemáticas, en alumnos del grado tercero de básica primaria de la Institución Educativa Neira (municipio de Neira, departamento de Caldas en Colombia), que mejorará su grado de satisfacción frente al estudio de esta área. Para este fin, se desarrolla una investigación con enfoque mixto, como lo sugieren Binotti et al. (2009) en la cual la evaluación cualitativa se soporta en los datos obtenidos por medio del componente cuantitativo. A pesar que diferentes investigadores han centrado sus esfuerzos en proponer modificaciones a los modelos educativos y a las estrategias didácticas existentes y sus derivaciones (Abrahamson, 2009), incluyendo en sus trabajos la neurociencia, la mayoría de ellos busca impactar positivamente el rendimiento académico de los estudiantes, es decir, se enfocan en el factor cognitivo. Este trabajo, por el contrario, pretende mejorar el grado de satisfacción de los estudiantes frente al estudio de esta área, dando prioridad al componente afectivo y tomando como punto de partida sus presaberes (Boaler, 2008).

\section{METODOLOGÍA}

Teniendo en cuenta que, el propósito general de esta investigación era diseñar e implementar un aula virtual para la enseñanza de las matemáticas en alumnos de tercer grado en la Institución Educativa Neira, para aumentar su grado de satisfacción frente al estudio de esta área y que se viera reflejado en la medida en que disfrutan las clases de esta asignatura y el agrado por la realización de las actividades planteadas en las mismas. El grado tercero de la Institución Educativa Neira cuenta con 20 estudiantes, de los cuales 8 eran niñas y 12 niños, con edades entre los ocho y diez años, pertenecientes a los estratos socioeconómicos 1, 2 y 3 . Se decidió implementar el aula virtual en este grado debido a que en este año escolar inicia la presentación de pruebas Saber, las cuales se enfocan principalmente en las áreas de lenguaje y matemáticas. Debido al lenguaje técnico presente en algunas de las preguntas se realizó acompañamiento a los estudiantes durante su aplicación, con el propósito de resolver dudas que pudieran surgir y, de esta manera, evitar respuestas erróneas o en blanco derivadas de una interpretación inadecuada.

El acuerdo al que se llegó con las directivas de la Institución Educativa Neira, fue que esta implementación sería una prueba piloto, y de acuerdo a los resultados obtenidos se realizaría en otros grados y otras asignaturas, gracias a la versatilidad del entorno virtual para realizar el ajuste de contenidos. Por lo anterior y buscando no entorpecer el normal funcionamiento de la jornada escolar se acordó que el curso se desarrollaría en la jornada contraria, en la sede José María Córdoba, lo cual redujo la población de aplicación a 11 estudiantes, quienes podían asistir en las tardes y no presentaban dificultades por cruces de horario con otras actividades, domicilio rural de difícil acceso o dinámicas especiales en sus hogares. El análisis, diseño e implementación de esta propuesta se desarrolló en los siguientes cinco pasos: Encuesta previa:; (2) Selección de contenidos; (3) Test de ideas previas de aritmética; (4) Test de ideas previas de pensamiento aleatorio; y (5) Diseño del aula virtual:

Paso 1. Encuesta previa: diseño y aplicación de una encuesta con una pregunta tipo Si/No y las demás tipo escala de Likert, en la cual se indagó lo que los estudiantes pensaban sobre las diferentes variables biológicas, sociales, económicas y personales que afectan el aprendizaje y el estudio de las matemáticas. La encuesta se aplicó a 11 estudiantes del grado tercero de primaria de la Institución Educativa Neira, durante el primer período académico del año 2017. Los interrogantes fueron: i) ¿Cree usted que a medida que crece, su desarrollo cerebral aumenta facilitando el aprendizaje? (Responda SI o NO). ii) De 1 a 5 , ¿cuánto cree que sus condiciones económicas y sociales en las que vive influyen en su proceso de aprendizaje? (1. No influyen- 5. Influyen completamente). iii) De 1 a 5 , ¿cuánto cree que el conocimiento del profesor en el área y los recursos de aula que utiliza influyen en su proceso de aprendizaje? De 1 a 5 , ¿cuánto cree que el conocimiento del profesor en el área y los recursos de aula que utiliza influyen en su proceso de aprendizaje? iv) De 1 a 5, ¿cuánto disfruta de las clases de matemáticas? (1. No las disfruta5. Las disfruta completamente). A partir de la quinta pregunta se respondía teniendo en cuenta el enunciado "En el momento de resolver un problema en el área de matemáticas, qué importancia cree que tiene cada uno de los siguientes procesos (califique de 1 a 5)": v) Identificar el problema y plantear las posibles soluciones. vi) Centrar la atención. vii) Administrar el tiempo disponible. viii) Controlar emociones como la ansiedad y la frustración. ix) Recordar información x) Revisar todos los pasos realizados, identificando los posibles errores y cómo corregirlos. 
Paso 2. Selección de contenidos: la selección de los contenidos propuestos y las actividades desarrolladas se realizaron a partir de la revisión del plan de estudios institucional de matemáticas del grado tercero y la discusión con la comunidad de aprendizaje responsable de esta materia sobre los temas que usualmente presentan mayores dificultades en este nivel, así como los obstáculos observados en el grupo donde se proyectó la implementación de la herramienta propuesta y el enfoque de las pruebas estandarizadas aplicadas en este grado. Se evaluaron las competencias en los procesos generales del aprendizaje tales como el razonamiento, la resolución y planteamiento de problemas; la modelación y la elaboración; y la comparación y ejercitación de procedimientos (Ministerio de Educación Nacional, 1998).

Paso 3. Test de ideas previas de aritmética: se aplicó a los estudiantes de grado tercero en la Institución Educativa Neira (municipio de Neira, Caldas en Colombia), el tema central de esta valoración fue la multiplicación, y en ella se propusieron diferentes ejercicios y problemas con el propósito de conocer de antemano las principales dificultades y fortalezas de los estudiantes en esta temática a partir de las cuales se identificaron y formularon tareas específicas que influyeran en el razonamiento particular de los estudiantes (Dyer y Sherin, 2015).

El test constó de cinco preguntas en las cuales se evaluaron las competencias en ejercitación, modelación, razonamiento y solución de problemas, en conceptos aritméticos asociados a la multiplicación: en la primera pregunta se evaluó el proceso general de la ejercitación y se buscó identificar si los estudiantes tenían claro el concepto de la multiplicación y sus componentes (factores y producto) La segunda pregunta, valoró las competencias en modelación, así como la capacidad para proponer opciones de factores para el mismo producto, para lo cual se requería tener el concepto de la multiplicación y utilizar adecuadamente sus propiedades, específicamente la conmutativa. La tercera pregunta, se enfocó al razonamiento y tuvo como objetivo observar la capacidad de análisis del estudiante al identificar el factor faltante dentro de una multiplicación en la que el resultado era conocido. En la cuarta pregunta se presentó la solución de problemas con el fin de observar la capacidad de los estudiantes para plantear operaciones que dieran solución a un problema y, posteriormente, resolverlas. Y, finalmente, en la quinta pregunta se buscó reconocer la capacidad del estudiante para identificar las equivalencias entre las representaciones horizontal y vertical de una multiplicación e identificar esta última como el primer paso para aplicar el algoritmo de esta operación.

Paso 4. Test de ideas previas de pensamiento aleatorio: esta valoración se encaminó hacia los conceptos de estadística relacionados con la agrupación, la simplificación, el análisis y la representación de datos en tablas de frecuencias y diagramas de barras. Los tres ejercicios planteados evaluaron las competencias en ejercitación y razonamiento. Inicialmente, se buscó determinar si los estudiantes comprendían la información presentada en las tablas de frecuencias y su capacidad para sintetizar estos datos a través de preguntas derivadas de ellas. En la segunda parte del test se indagó sobre la facultad de los estudiantes para sintetizar y expresar la información presentada en un diagrama de barras.

Paso 5. Diseño del aula virtual: se construyó e implementó un aula virtual en la plataforma Moodle proporcionada de forma gratuita por el sitio MilAulas (2011), en la cual se creó el curso Matemáticas 3, que contenía dos secciones: Multiplicación; y Tablas de frecuencias, moda y diagramas. Las actividades propuestas en el aula virtual buscaron, primero, reforzar el concepto de la multiplicación, para que el estudiante la reconociera como una suma abreviada; segundo, que el estudiante identificara los elementos y las propiedades de esta operación; y, tercero, fortalecer las competencias de razonamiento y ejercitación mediante la modelación y resolución de problemas en los que se buscó el planteamiento y resolución de multiplicaciones, a partir del reconocimiento del aprendizaje como un proceso individual directamente relacionado con los recursos cognitivos de cada estudiante (Vidal et al., 2015). El primer recurso de aula virtual se creó con la herramienta Powtoon (Zaturansky, D. y Spitalnik, I., 2011) (Ver Figura 1) consistente en un video introductorio, en el cual se informó a los estudiantes sobre la finalidad del curso, las secciones que contenía y los temas seleccionados para ser desarrollados en cada sección, existía una ruta sugerida para realizar las actividades, pero se permitió a los estudiantes seguir sus propios ritmos y preferencias de exploración.

Posteriormente, en la sección de aritmética, se adjuntó el test de ideas previas que buscó identificar los obstáculos de aprendizaje del grupo objetivo, con el propósito de enfocar adecuadamente las actividades de esta sección. Debido a lo anterior decidió realizar una presentación que incluyó la conceptualización, los componentes y las propiedades de la multiplicación, así como el planteamiento de las soluciones a los problemas relacionados con la cotidianidad de los estudiantes que involucraron realizar multiplicaciones. Luego, se adjuntó un enlace a una herramienta de práctica proporcionada por el sitio (ThatQuiz, 2017) que ofrecía multiplicaciones de diferentes grados de dificultad generadas de manera aleatoria y que se configuró de forma tal que permitía al estudiante verificar si la respuesta dada era correcta y, de no ser así, mostraba el resultado apropiado, es decir, el estudiante verificó por sí mismo sus aciertos y fallos en las actividades resueltas y propuso acciones correctivas para solucionar sus desaciertos. 
Como evaluación y cierre de esta sección, se ofreció un cuestionario con preguntas de arrastrar y soltar, emparejamiento, selección múltiple, calculadas y numéricas, que incluían conceptos y problemas asociados a la multiplicación, los problemas incluían circunstancias presentes en la cotidianidad de su entorno. El cuestionario fue diseñado de forma tal que en el momento en que se resolvía una pregunta, sí existía algún error se mostraba la respuesta correcta, permitiendo al estudiante que a partir de la consciencia de su propio aprendizaje evidenciara por sí mismo en que parte del proceso había fallado, y pensara en qué medidas debía tomar para corregirlo y así poder pasar a la siguiente pregunta, sin que el número de intentos para responder correctamente afectara su calificación. De esta manera se incentivó el uso del entorno virtual, a la vez que se buscó potenciar el aprendizaje autorregulado, la autonomía y el automonitoreo en el desarrollo de las actividades.

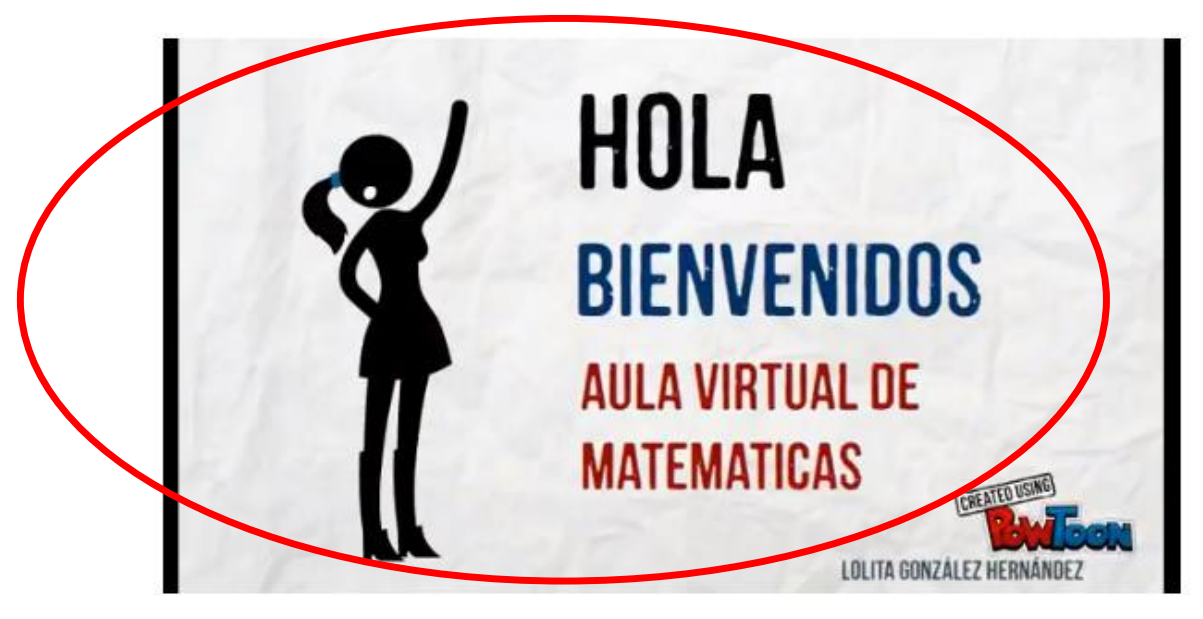

Video construido utilizando Powtoon

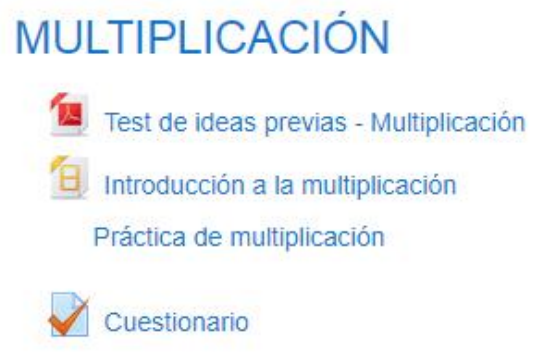

Test de presaberes

Conceptualización

Enlace al sitio thatquiz para practicar el algoritmo de la multiplicación

Cuestionario construido a partir del banco de 40 preguntas

Fig. 1: Aula virtual: Video de bienvenida (Zaturansky, D. y Spitalnik, I., 2011) y actividades de Multiplicación. Imagen adaptada de MilAlulas (2011)

Para identificar los presaberes en la sección de estadística, al igual que en la de multiplicación se inició con un test de ideas previas, para identificar los preconceptos de los estudiantes. A partir de los hallazgos se determinó realizar en primer lugar una inducción a Microsoft Excel, en la cual se dieron las instrucciones básicas de funcionamiento de esta herramienta ofimática, sus características y, la creación y manipulación de diagramas de barras. Posteriormente, se propusieron dos talleres que contenían problemas relacionados con su cotidianidad, que incluyen la construcción y el análisis de gráficos estadísticos. En el aula virtual se pudieron observar, además, los talleres resueltos con observaciones y aclaraciones, con la intención de permitir a los estudiantes identificar sus errores puntuales en la resolución de las actividades si los tenían y corregirlos para obtener el resultado correcto.

Paso 6. Implementación del aula virtual: se realizó en la sala de sistemas de la Institución Educativa Neira, sede José María Córdoba, durante 10 sesiones de 2 horas cada una. Las dos sesiones iniciales se destinaron a la introducción y el registro de los usuarios en la plataforma Moodle, así como para la entrega de las instrucciones básicas de ingreso y funcionamiento y la aplicación de los test de ideas previas. Las siguientes tres sesiones se utilizaron para el desarrollo de la sección de multiplicación, dos sesiones más se destinaron a la inducción y exploración de Microsoft Excel, con el propósito de facilitar el posterior desarrollo de los temas propuestos en la sección tablas de frecuencia, moda y diagramas de barras, en las dos siguientes sesiones; y, finalmente, el último encuentro se dedicó a la retroalimentación general del curso y la aplicación de la encuesta final de satisfacción.

Paso 7. Encuesta final de satisfacción: se aplicó a los estudiantes participantes en el curso y constó de ocho preguntas, las cuales procuraron indagar sobre el grado de satisfacción de los estudiantes frente al estudio de las matemáticas y la acogida de la herramienta propuesta. Por esta razón, se incluyeron 
preguntas relacionadas a las estrategias utilizadas durante el curso, así como la pregunta utilizada en la encuesta inicial (Ver Paso 1) para medir el grado de satisfacción frente al estudio de las matemáticas. Los interrogantes fueron los siguientes: i) ¿Considera que el curso lo ha ayudado a comprender mejor los temas vistos? ii) ¿Considera que la forma en qué se dictaron las clases fueron de su agrado? iii) ¿Se siente cómodo identificando y corrigiendo usted mismo las actividades propuestas? iv) Revisar usted mismo las respuestas correctas y compararlas con las suyas para evaluar su proceso, le parece agradable? v) Revisar usted mismo las respuestas correctas y compararlas con las suyas para evaluar su proceso, le ha hecho ganar confianza en el aprendizaje de las matemáticas? vi) ¿Considera que fue agradable la forma en que se mostraron las matemáticas en el curso? vii) Luego de asistir al curso, ¿siente que las matemáticas le agradan más? viii) De 1 a 5, ¿cuánto disfruta de las clases de matemáticas? (1. No las disfruta- 5 . Las disfruta completamente).

\section{RESULTADOS}

A partir del planteamiento del objetivo general, a continuación, se presentan los resultados de cada uno de los pasos propuestos e implementados en el desarrollo metodológico:

Paso 1. Encuesta previa: una vez se realizó la aplicación de este instrumento se pudo identificar que sólo el $64 \%$ de los estudiantes encuestados dieron la máxima calificación a la pregunta sobre si disfrutaban las clases de matemáticas. Por otro lado, variables como las condiciones socio-económicas, los recursos del aula y el conocimiento específico de los profesores les parecieron poco influyentes en su proceso de aprendizaje. Adicionalmente se evidenció que en el momento de resolver un problema en el área de matemáticas lo que tuvo mayor peso para los estudiantes fue la identificación del problema y el planteamiento de las posibles soluciones, a lo que todos dieron la máxima calificación. Así mismo, el 91\% de los encuestados evaluaron con el máximo valor en la escala la importancia de revisar todos los pasos realizados, identificando los posibles errores y corrigiéndolos. Con base en lo anterior se intuyó que la herramienta debía centrarse en potenciar el razonamiento y la función ejecutiva del automonitoreo, para mejorar la percepción de los estudiantes frente a las matemáticas y de esta forma aumentar el grado de satisfacción.

Paso 2. Selección de contenidos: se eligieron como temas la multiplicación en la sección de aritmética y los diagramas de barras junto con las tablas de frecuencias y la moda en la de estadística. Además, se decidió realizar un test de ideas previas para cada sección: el primero enfocado al pensamiento numérico, específicamente en el tema de la multiplicación y, el segundo, en el pensamiento aleatorio para el tema de estadística.

Paso 3. Test de ideas previas de aritmética: entre los obstáculos de aprendizaje se encontraron la falta de dominio del concepto de la multiplicación, así como la dificultad para identificar sus componentes y aplicar sus propiedades; y el más destacado fue la imposibilidad para plantear y resolver problemas cuando la operación a realizar era una multiplicación. Adicionalmente, este test permitió identificar que más de un $91 \%$ de la población evaluada no realizaba una asociación correcta entre la representación horizontal y la vertical de la multiplicación, por lo cual no llegaron a un resultado correcto, debido a que, la representación vertical es el primer paso para aplicar el algoritmo de la multiplicación en operaciones con números de varios dígitos.

Paso 4. Test de ideas previas de pensamiento aleatorio: esta valoración arrojó como resultado que los estudiantes poseían buenas bases en estos temas y mostraban facilidad para sintetizar conjuntos de datos presentados en tablas de frecuencias y diagramas de barras. Esta fortaleza encontrada en los estudiantes, permitió que, en el diseño del aula virtual, se consideraran ejercicios encaminados a afianzar estos conceptos, contextualizar su aplicación y aplicarlos utilizando las TIC. En este caso se empleó la herramienta Microsoft Excel con el fin de mostrar a los estudiantes cómo pueden llegar a los mismos resultados con el uso de esta aplicación.

Paso 5. Aula virtual: en la sección de aritmética se adjuntó el test de ideas previas (Ver paso 3) y a partir de los obstáculos de aprendizaje detectados, se construyó un banco de 40 preguntas de diferentes tipos: arrastrar y soltar, calculada, numérica, de opción múltiple, etc. orientadas a la conceptualización de la multiplicación, la identificación de sus componentes y la correcta aplicación de las propiedades de esta operación, así mismo algunas incluían problemas relacionados con la cotidianidad de los estudiantes, los cuales para ser resueltos requerían realizar multiplicaciones. La práctica de multiplicación y el cuestionario tuvieron aceptación entre los estudiantes, especialmente por el hecho de permitirles observar el resultado correcto de cada pregunta y compararlo con la solución obtenida por ellos. 
En la sección de estadística, se decidió iniciar con un test de ideas previas y, a partir de los resultados obtenidos se formularon dos talleres que incluyeron la construcción y el análisis de gráficos estadísticos, además del manejo de diagramas de barras en la hoja de cálculo de Excel. Los problemas propuestos presentaron situaciones cercanas a la cotidianidad de los estudiantes. Adicionalmente se adjuntaron los talleres resueltos, de esta forma los estudiantes tuvieron acceso a ellos durante toda la implementación, lo que les permitió identificar y corregir de forma autónoma sus equivocaciones y las implicaciones de éstas sobre el resultado final.



Fig. 2: Aula virtual: Primer taller de estadística. Imagen adaptada de MilAlulas (2011)

Paso 6. Implementación del aula virtual: aunque el grupo inicialmente estaba compuesto por 20 estudiantes, se implementó la herramienta con 11 de ellos, quienes accedieron a realizar el curso en jornada contraria, para no interferir en su cronograma escolar. La utilización de las TIC como herramienta pedagógica, motivó significativamente a los estudiantes a participar de las actividades propuestas en el aula virtual, lo anterior se comprobó en la actitud mostrada hacía el curso y la forma en que fue desarrollado. Se evidenció, además, que los estudiantes presentaron problemas de conceptualización, un ejemplo claro de esto fue el hecho que algunos de ellos a pesar de haber memorizado las tablas de multiplicar, no tenían claro el concepto de multiplicación, su significado y su aplicación en la cotidianidad y, por esta razón, mostraron dificultad para resolver los problemas matemáticos planteados, lo que aumentaba su grado de frustración y desagrado frente a la asignatura.

Fue necesario realizar dos sesiones introductorias, donde además de explicar los contenidos a desarrollar se dieron las instrucciones de manejo del explorador, se entregó la información de acceso a la plataforma Moodle y se brindaron las directrices para ir desarrollando las actividades de acuerdo a la ruta planteada, a pesar de lo anterior se permitió que cada estudiante de manera autónoma escogiera los tiempos y la secuencia de las tareas, de acuerdo a sus preferencias y recursos cognitivos, lo cual les generó en los estudiantes satisfacción y seguridad debido a la inexistencia de un registro de errores o calificaciones, contrariamente cada uno de ellos era responsable de autoevaluarse e identificar sus desaciertos, con el propósito de corregirlos y conseguir el resultado esperado, potenciando así la función neurocognitiva del automonitoreo (del-Buey et al., 2007).

Paso 7. Encuesta final de satisfacción: al finalizar la implementación del aula virtual, se aplicó una encuesta de satisfacción, en la cual un 100\% de los encuestados afirmaron que este curso les ayudó a comprender mejor los temas vistos y, que luego de asistir, las matemáticas le agradan más. Así mismo, un 91\% considera que fue agradable la forma en que se mostraron las matemáticas en el curso y, además, se sienten cómodos identificando y corrigiendo ellos mismos las actividades propuestas. Los resultados de las preguntas tipo $\mathrm{Si}$ o No se pueden observar en la figura 3 y los de la octava pregunta tipo Likert se muestran en la figura 4.

Paso 8. Comparación del grado de satisfacción frente al estudio de las matemáticas: con el propósito de medir el impacto en esta variable se compararon los resultados de la pregunta vi) de la encuesta previa y la pregunta viii) de la encuesta final de satisfacción: viii) De 1 a 5 , ¿cuánto disfruta de las clases de matemáticas? (1.No las disfruta- 5. Las disfruta completamente). En la primera encuesta el $36 \%$ de los encuestados respondió que no las disfrutaba posteriormente se evidenció que a partir de la aplicación de la herramienta el porcentaje de estudiantes que las disfrutaba completamente aumentó del $55 \%$ al $91 \%$, es decir se obtuvo un mejoramiento del $36 \%$ en el grado de satisfacción de los estudiantes frente al estudio de las matemáticas, como se observa en la figura 4. 


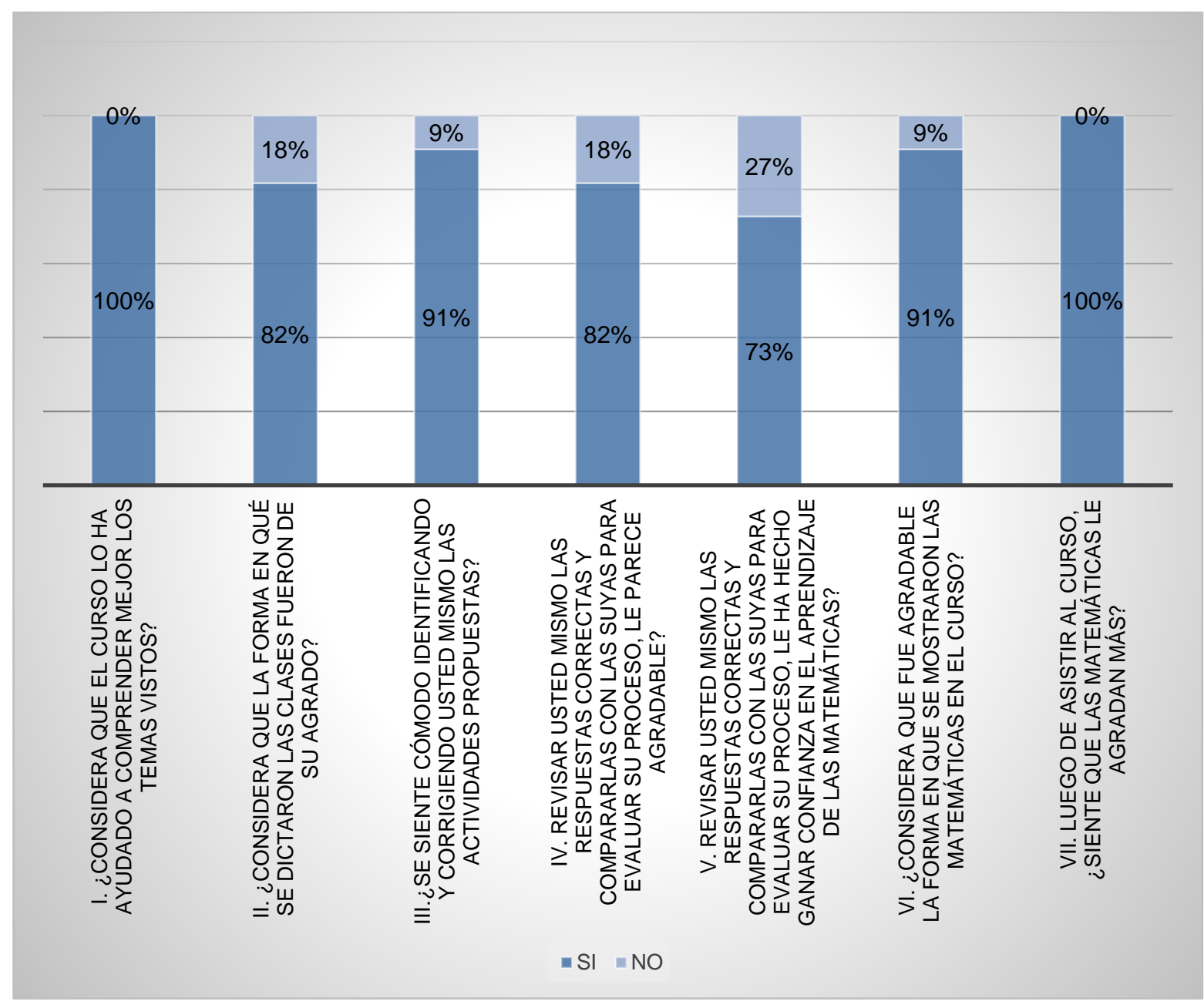

Fig. 3: Resultados de la encuesta de satisfacción (Preguntas i - vii)



Fig. 4: Comparación del grado de satisfacción frente al estudio de las matemáticas.

\section{DISCUSIÓN}

Diferentes investigaciones plantean estrategias de aula para el fortalecimiento de algunas funciones neurocognitivas (Poenitz, 2015) reconociendo su importancia en los procesos de enseñanza-aprendizaje. En ocasiones, estas investigaciones hacen uso de bancos preguntas y test que a menudo se incluyen sólo 
de manera parcial en el estudio, debido a que una vez se realiza la implementación del estudio, se suelen presentar cambios en los objetivos o en las prioridades iniciales. Debido a lo anterior se resalta la importancia de soportar la evaluación cualitativa, con los datos obtenidos a través de la metodología cuantitativa (Binotti et al., 2009).

Este trabajo proporciona una estrategia de aula que permite el desarrollo de los contenidos propuestos, a través de herramientas que conlleven a la potencialización de la función neurocognitiva del automonitoreo en cada estudiante, la mayoría de estudios que involucran las funciones ejecutivas, se enfocan en evaluar su incidencia sobre el rendimiento académico (Barrera et al., 2017;Castillo-Parra et al., 2009) y otros en mejorarlo (Posada-Ortíz, 2012; Basto-Alvarado, 2017; Barrera et al.), en esta investigación por el contrario, se busca mejorar el grado de satisfacción frente al estudio de las matemáticas, es decir, se centra la atención en el factor afectivo más que en el cognitivo. Lo anterior toma especial relevancia cuando se tiene en cuenta que uno de los principales obstáculos que presenta el estudio de las matemáticas es el hecho de ser una materia que despierta poco aprecio e interés en los estudiantes (Farías y Pérez, 2010).

La estrategia diseñada en este trabajo de investigación está enmarcada en las tendencias educativas actuales en las que el nuevo enfoque pedagógico incorpora herramientas digitales, en las cuales el docente es el encargado de monitorear e impartir instrucciones dentro de los procesos de aprendizaje, a la vez que reconoce el papel activo del estudiante, con especial atención sobre la reflexión, la metacognición y la autorregulación del aprendizaje (Chaves y Trujillo, 2015). La metodología empleada buscó el aprendizaje a través del reconocimiento y la corrección de los errores a partir de la comparación autónoma de los resultados, los procedimientos ejecutados y las respuestas propias.

Por otro lado, no es de extrañar que en el presente apogeo informático, diferentes autores planteen metodologías de enseñanza basadas en las TIC (Basantes et al., 2017; Valenzuela-Zambrano y PérezVillalobos, 2013)las cuales permiten la integración de diferentes disciplinas (Televantou et al., 2015) a partir de la simbiosis entre la didáctica y la tecnología. En este trabajo en particular, la utilización de las TIC, y en específico del aula virtual, no solo amplía la cobertura de la estrategia, también facilita la incorporación y actualización de cursos y actividades, permite llevar un registro constante de los avances de cada estudiante, y facilita la identificación de dificultades y oportunidades que pueden ser aprovechadas en futuras propuestas.

\section{DISCUSION FINAL}

Este trabajo permitió evidenciar que es conveniente enfocar la enseñanza de las matemáticas a fortalecer la conceptualización, la contextualización de los contenidos y la resolución de problemas, con la intención de invitar al estudiante a construir su propio conocimiento, trazando una ruta pedagógica y motivándolos a recorrerla a su propio ritmo y de acuerdo a sus recursos cognitivos. Si bien el aula virtual diseñada fue aplicada para un caso particular, puede ser utilizada en cualquier grado y materia, modificando los contenidos y niveles de dificultad de las actividades planteadas, explotando la versatilidad del entorno virtual. Aunque la implementación del aula virtual se realizó con solamente 11 estudiantes, es de resaltar que se hizo como una prueba piloto, según lo acordado con las directivas de la Institución Educativa Neira y debido a la respuesta positiva de la comunidad educativa frente al desarrollo de esta herramienta, es posible incorporarla en el Proyecto Educativo Institucional en diferentes áreas y grados, modificando los contenidos, pero conservando la metodología de aprendizaje autorregulado a partir del error.

La aplicación del aula virtual diseñada mejoró la percepción y aumentó el grado de satisfacción frente al estudio de las matemáticas de los estudiantes del grado tercero de la institución educativa Neira que tomaron el curso en un 36\%. Los resultados se obtuvieron mediante la técnica de encuestas, aplicadas a los estudiantes con quienes se implementó la herramienta. En las cuales se buscó identificar la influencia de algunas variables biológicas, sociales, económicas y personales en el aprendizaje de las matemáticas, así como medir el aumento en el grado de satisfacción de los estudiantes frente al estudio de las matemáticas, entendido como la alegría relacionada con el cumplimiento de las actividades propuestas y los resultados obtenidos (Kangas et al., 2017), reflejados en la medida en que disfrutan las clases de esta asignatura. Los resultados indicaron que los temas tratados fueron del agrado de los estudiantes, también manifestaron que el hecho de revisar los procedimientos, así como encontrar de manera autónoma sus errores y poder aplicarlos especialmente en los problemas relacionados con su contexto social y su cotidianidad aumentaron su agrado hacia el aprendizaje de las matemáticas.

El presente trabajo puede constituirse como un antecedente para el desarrollo de futuras herramientas encaminadas al fortalecimiento de la función neurocognitiva del automonitoreo a partir de la consciencia del propio aprendizaje, la detección de errores a partir de la comparación con los procedimientos desarrollados adecuadamente y el planteamiento de acciones que conlleven a posibles soluciones. 


\section{CONCLUSIONES}

De acuerdo al trabajo presentado y a los resultados obtenidos, se pueden plantear las siguientes conclusiones principales:

1.- En el caso específico de la enseñanza-aprendizaje de las matemáticas se debe prestar especial atención a los recursos cognitivos de cada estudiante, involucrarlo en la construcción de su propio conocimiento y potenciar su razonamiento a través del planteamiento y la resolución de problemas asociados a su cotidianidad.

2.- En el momento de diseñar e implementar esta herramienta pedagógica fue determinante indagar los presaberes del grupo objetivo, así como las variables biológicas, sociales y económicas que consideraban influyentes en su proceso de aprendizaje.

3.- El uso de las TIC incentivó notablemente la participación de los estudiantes, lo cual se evidenció en su interés por desarrollar las diferentes actividades propuestas en el aula virtual. Así mismo, la versatilidad del aula virtual, facilitó la incorporación, modificación y actualización de contenidos.

\section{AGRADECIMIENTOS}

Los autores agradecen al proyecto de la Secretaria de Educación Departamental de Caldas - Colombia: "Estudios de maestría mediante becas a docentes de los establecimientos educativos oficiales de los municipios no certificados en el departamento de Caldas". Al apoyo incondicional recibido por la comunidad de la Institución Educativa Neira, y la colaboración de la U. Nacional de Colombia, Sede Manizales.

\section{REFERENCIAS}

Abrahamson, D., Embodied design: Constructing Means for Constructing Meaning, doi: 10.1007/s10649-008-9137-1, Educational Studies in Mathematics, 70(1), 27-47 (2009)

Barrera D., C. Ordúz y M. Zambrano, Programa Grupal Lúdico-Motriz y Cognitivo-Emocional Pensamos, Sentimos, Aprendemos como Apoyo en el Fortalecimiento de Funciones Ejecutivas en niños de 5 a 6 Años (2017)

Basantes, A.V., M.E. Naranjo, M.C. Gallegos y N.M. Benítez, Los Dispositivos Móviles en el Proceso de Aprendizaje de la Facultad de Educación Ciencia y Tecnología de la Universidad Técnica del Norte de Ecuador, doi: 10.4067/S071850062017000200009, Formación Universitaria, 10, 79-88 (2017)

Basto-Alvarado, L., Pensamiento Variacional en Estudiantes de Grado Noveno del Instituto Integrado de Enseñanza Media Comercial San José de Suaita (2017)

Binotti, P., D. Spina, M. de la Barrera y D. Donolo, Funciones Ejecutivas y Aprendizaje en el Envejecimiento Normal, Estimulación Cognitiva desde una Mirada Psicopedagógica, Revista Chilena de Neuropsicología, 4(2), 119-126 (2009)

Bishop, A.J., Enculturación Matemática: la Educación Matemática desde una Perspectiva Cultural, Temas de Educación, Paidós (1999)

Boaler, J., When Politics Took the Place of Inquiry: A Response to the National Mathematics Advisory Panel's Review of Instructional Practices, doi: 10.3102/0013189x08327998, Educational Researcher, 37(9), 588-594 (2008)

Castillo-Parra, G., E. Gómez y F. Ostrosky-Solís, Relación entre las funciones cognitivas y el nivel de rendimiento académico en niños, Revista Neuropsicología, Neuropsiquiatría y Neurociencias, 9(1), 41-54 (2009)

Castro, M., La Ciencia es una Forma Cultural Necesaria para Vivir, Entrevista a Neus Sanmartí, Especialista en Didáctica de las Ciencias (2005)

Chaves, E. y J.M. Trujillo, Autorregulación del Aprendizaje en Entornos Personales de Aprendizaje en el Grado de Educación Primaria de la Universidad de Granada, España, doi: 10.4067/S0718-50062015000400008, Formación Universitaria, 8(4), 63-76 (2015)

Cohen, A. y O. Baruth, Personality, Learning, and Satisfaction in Fully Online Academic Courses, doi: 10.1016/j.chb.2017.02.030, Computers in Human Behavior (2017)

Contreras, K., C. Caballero, J. Palacio y M. Pérez, Factores Asociados al Fracaso Académico en Estudiantes Universitarios de Barranquilla (Colombia), ISSN: 2011-7485, Psicologia desde El Caribe, U. del Norte, 22, 110-135 (2008)

Correa, C., M. Fernández-Alcántara y otros tres autores, Efectos de un Programa de Estimulación de las Funciones Ejecutivas en Niños con Dificultades de Aprendizaje, doi:10.1080/02109395.2017.1295576, Estudios de Psicología, 115 (2017)

del-Buey, F., M.E. Martín-Palacio, F. Camarero-Suárez y C. Sáez-Navarro, Procesos Metacognitivos: Estrategias y Técnicas (2007)

Dyer, E.B. y M.G. Sherin, Instructional Reasoning about Interpretations of Student Thinking that Supports Responsive Teaching in Secondary Mathematics, doi: 10.1007/s11858-015-0740-1, ZDM Mathematics Education, 48(1), 1-14 (2015) 
Ernest, P., The Unit of Analysis in Mathematics Education: Bridging the Political-Technical Divide?, doi:10.1007/s10649016-9689-4,The Educational Studies in Mathematics, 92(1), 37-58 (2016)

Esteban-Albert, M. y M. Zapata-Ros, Estrategias de Aprendizaje y eLearning. Un Apunte para la Fundamentación del Diseño Educativo en los Entornos Virtuales de Aprendizaje, doi: http://dx.doi.org/10.6018/red/50/15, RED, Revista de Educación a Distancia (2016)

Falco, M., Reconsiderando las Prácticas Educativas: TICs en el Proceso de Enseñanza-Aprendizaje, doi: 10.15366/tp2017.29.002, Tendencias Pedagógicas, 29, 59-76 (2017)

Farias, D. y J. Pérez, Motivación en la Enseñanza de las Matemáticas y la Administración, doi: 10.4067/S071850062010000600005, Formación Universitaria, 3(6), 33-40 (2010)

Hernández, C.A, C. Cárdenas, P. Romero y M. Hernández, Los Padres de Familia y el Logro Académico de los Adolescentes de una Secundaria en Milpa Alta, Ciudad de México, doi:10.4067/S0718-07642017000300013, Información Tecnológica, 28(3), 119-128 (2017)

Julisa, V., Biología y Educación: un Nuevo Punto de Encuentro, doi:10.5377/unahinnov.v0i3.2384, UNAH INNOV@, 3, 32-38 (2014)

Kangas, M., P. Siklander, J. Randolph y H. Ruokamo, Teachers' Engagement and Students' Satisfaction with a Playful Learning Environment, doi: 10.1016/j.tate.2016.12.018, Teaching and Teacher Education, 63, 274-284 (2017)

Kline, M. A., How to Learn About Teaching : An Evolutionary Framework for the Study of Teaching Behavior in Humans and Other Animals, doi:10.1017/S0140525X140000901-71, Behavioral and Brain Sciences, 1-71 (2015)

Martin, A.J., Enhancing Student Motivation and Engagement: The Effects of a Multidimensional Intervention, doi: 10.1016/j.cedpsych.2006.11.003, Contemporary Educational Psychology, 33(2), 239-269 (2008)

Melo-Solarte, D.S. y P. Díaz, El Aprendizaje Afectivo y la Gamificación en Escenarios de Educación Virtual, doi:http://dx.doi.org/10.4067/S0718-07642018000300237, Información Tecnológica, 29(3), 237-248 (2018)

MilAulas, https://www.milaulas.com, acceso: febrero 28 de 2018 (2011)

Ministerio de Educación Nacional, Lineamientos Curriculares Matemáticas (1998)

Ministerio de Educación Nacional, Programa Todos a Aprender: Para la Transformación de la Calidad Educativa (2012)

Poenitz, A., Estrategias para la estimulación de las Funciones Cognitivas en el Aula, I Congreso Dominicano de Neurociencia, 1-4, Santo Domingo, República Dominicana, 28-30 de Octubre (2015)

Posada-Ortíz, D., Implementacion de Estrategias Constructivistas en la Enseñanza del Algebra, que Fomenten el Desarrollo de la Funcion Neurocognitiva Automonitoreo, como un Estudio de Caso en la Sección 20 del Grado Octavo de la Educacion Básica de la I. E. INEM José Félix (2012)

Ramírez-Díaz, J., Satisfacción de Estudiantes de Secundaria Nocturna y su Incidencia en el Abandono Escolar, doi:http://dx.doi.org/10.15359/ree.22-1.14, Revista Electrónica Educare (2018)

Sastre-Riba, S., Condiciones Tempranas del Desarrollo y el Aprendizaje: el Papel de las Funciones Ejecutivas, Simposio Satélite: Dificultades del Aprendizaje, 42, 143-151 (2006)

Shulman, L.S., Knowledge and Teaching: Foundations of New Reform, doi: https://doi.org/10.17763/haer.57.1.j463w79r56455411, Harvard Educational Review (1987)

Televantou, I., H. Marsh y otros cuatro autores, Phantom Effects in School Composition Research: Consequences of Failure to Control Biases due to Measurement Error in Traditional Multilevel Models, doi: 10.1080/09243453.2013.871302, School Effectiveness and School Improvement, 26(1), 75-101 (2015)

ThatQuiz, https://www.thatquiz.org, acceso: febrero 28 de 2018 (2017)

Tirapu-Ustárroz, J., J. M. Muñoz-Céspedes y C. Pelegrín-Valero, Funciones Ejecutivas: Necesidad de una Integración Conceptual, Revista de Neurología, 34(7), 673-685 (2002)

Valenzuela-Zambrano, B. y M.V. Pérez-Villalobos, Aprendizaje autorregulado a través de la plataforma virtual Moodle, Educación y Educadores, 16(1), 66-79 (2013)

Vidal, C.L., C. Cabezas, J.H. Parra y L.P. López, Experiencias Prácticas con el Uso del Lenguaje de Programación Scratch para Desarrollar el Pensamiento Algorítmico de Estudiantes en Chile, doi: 10.4067/S071850062015000400004, Formación Universitaria, 8(4), 23-32 (2015)

Villarruel, M., La Práctica Educativa del Maestro Mediador, Revista Iberoamericana de Educación, 50(3), 2-12 (2009)

Vygotsky, L.S., Interaction Between Learning and Development, Mind and Society, doi: 10.1016/S0006-3495(96)795723, 29-36 (1978a)

Vygotsky, L.S., Tool and Symbol in Child Development Chapter, Mind and Society (1978b)

Zaturansky, D. e I. Spitalnik, PowToon, https://www.powtoon.com, acceso: febrero 28 de 2018 (2011)

Zimmerman, B.J. y D.H. Schunk, Handbook of Self-Regulation of Learning and Performance, doi: 10.4324/9780203839010.ch1, Educational Psychology handbook series, Routledge (2017) 
